\title{
Three-dimensional simulations of the expanding remnant of SN 1987A
}

\author{
Toby Potter ${ }^{1}$, Lister Staveley-Smith ${ }^{1}$, John Kirk ${ }^{2}$, Brian Reville ${ }^{3}$, \\ Geoff Bicknell $^{4}$, Ralph Sutherland ${ }^{4}$, Alexander Wagner ${ }^{4}$, \\ and Giovanna Zanardo ${ }^{1}$ \\ ${ }^{1}$ International Centre for Radio Astronomy Research \\ M468, The University of Western Australia \\ 35 Stirling Hwy, Crawley \\ Western Australia, 6009 \\ ${ }^{2}$ Max-Planck-Institut für Kernphysik, Heidelberg, Germany \\ ${ }^{3}$ Department of Physics, University of Oxford \\ ${ }^{4}$ Research School of Astronomy and Astrophysics, \\ Australian National University \\ email: tobympotter@gmail.com
}

\begin{abstract}
SNR 1987A is the expanding remnant from the brightest supernova since the invention of the telescope. The remnant has been monitored extensively in the radio at variety of wavelengths and provides a wealth of data on which to base a simulation. Questions to be answered include estimating the efficiency of particle acceleration at shock fronts, determining the cause of the one-sided radio morphology for SNR 1987A and investigating the gas properties of the pre-supernova environment. We attempt to address these questions using a fully three-dimensional model of SNR 1987A.
\end{abstract}

Keywords. Supernovae, SN 1987A, particle acceleration, three-dimensional, simulation

\section{Our model}

We use the hydrodynamics code FLASH (Fryxell et al. 2000) to simulate SNR 1987A in a $256^{3}$ grid. We derive radio emission in post-processing by using semi-analytic models of sub-Diffusive Shock Acceleration (Kirk et al. 1996) to place inverse power-law phasespace distributions of electrons at the shock front. The magnetic field is assumed to be amplified at the shock using physics from Bell (2004). Both magnetic field and electron distributions are advected downstream using the velocity field from the hydrodynamics simulation. Features of the model include a custom Truelove and McKee (1999) progenitor with $\mathrm{n}=9, E=1.5 \times 10^{44} \mathrm{~J}$ and $M=10 M_{\odot}$; a free blue supergiant (BSG) wind; a shocked BSG wind, a central equatorial ring and the hourglass. Turbulence was excited in the expanding shock by perturbing the initial density by a random number chosen from a lognormal distribution with mean 1.0 and variance 5.0 (Sutherland and Bicknell 2007).

\section{Results and conclusions of the study}

Optimal fits to the asymmetric radio morphology were obtained if 1.55 times more kinetic energy was placed in the eastern hemisphere of the progenitor. The best-fit radius for the free BSG wind was $3.2 \times 10^{15} \mathrm{~m}\left(0.43^{\prime \prime}\right)$ in the equatorial plane and $3.78 \times 10^{15}$ $\mathrm{m}\left(0.51^{\prime \prime}\right)$ in the polar direction. For the HII region the optimal half-opening angle was $\theta_{\text {ho }}=15 \pm 5^{\circ}$. Fits to the radius (see Figure 1) yield a peak HII region particle density of 

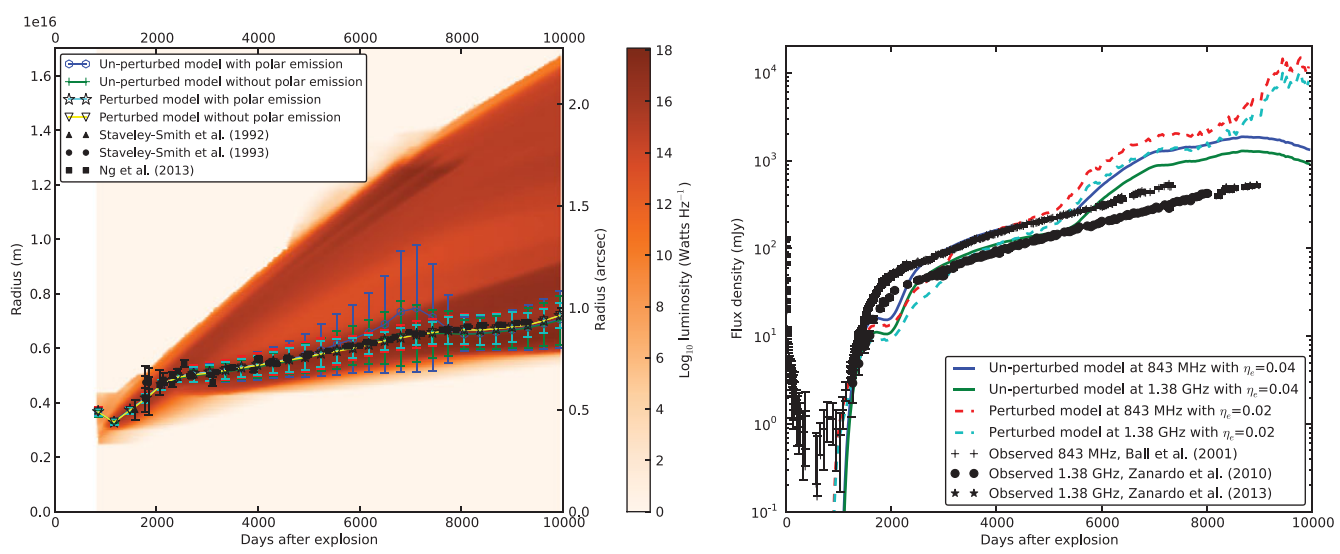

Figure 1. Radius and flux density from simulations of SN 1987A. The red background on the left is the time-varying radial distribution of simulated radio emission. Overlaid is the expectation of radius with error bars derived from expectation of variance. On the right the simulated flux density has been scaled by an injection efficiency $n_{e}$ in the range $(2-4 \%)$.

$(7.11 \pm 1.78) \times 10^{7} \mathrm{~m}^{-3}$, given an abundance of 3.5 particles per hydrogen atom. These results are consistent to within errors of Zhekov (2010).

For the equatorial ring we used a thin crescent torus (Plait et al. 1995), with a peak particle density of $8.0 \times 10^{8} \mathrm{~m}^{-3}$ for the smooth component. The torus was azimuthally interspersed with 21 Gaussian clouds (Dewey et al. 2012 ) of radius $2.25 \times 10^{14} \mathrm{~m}\left(0.03^{\prime \prime}\right)$, and a peak particle density of $3.14 \times 10^{10} \mathrm{~m}^{-3}$, consistent with Mattila et al. (2010). Radius measurements for both perturbed and unperturbed models agree well with the observational data from $\mathrm{Ng}$ et al. $(2008,2013)$ (see Figure 1). The simulations correctly model the apparent slowing in shockwave velocity seen around day 7500, and predict an accelerating expansion after day 9500. Flux density measurements (see Figure 1) from both un-perturbed and perturbed models show that the efficiency of particle acceleration for electrons is between 2 and $4 \%$. Predicted radio images show that the asymmetric radio morphology will reverse by day 10000 as the shock leaves the eastern lobe first.

\section{References}

Bell, A. R. 2004, MNRAS, 353, 550-558

Dewey, D., Dwarkadas, V. V., Haberl, F., Sturm, R., \& and Canizares, C. R. 2012, ApJ, 752, 103

Fryxell, B., Olson, K., Ricker, P., Timmes, F. X., Zingale, M., Lamb, D. Q., MacNeice, P., Rosner, R., Truran, J. W., \& Tufo, H. 2000, ApJS, 131, 273

Kirk, J. G., Duffy, P., \& Gallant, Y. A. 1996, A\&A A, 314, 1010-1016

Mattila, S., Lundqvist, P., Groningsson, P., Meikle, P., Stathakis, R., Fransson, C., \& Cannon, R. 2010, ApJ, 717, 1140-1156

Ng, C. Y., Gaensler, B. M., Staveley-Smith, L., Manchester, R. N., Kesteven, M. J., Ball, L., \& Tzioumis, A. K. 2008, ApJ, 684, 481-497

$\mathrm{Ng}, \mathrm{C} . \mathrm{Y}$. , et al. 2013 in-preparation

Plait, P. C., Lundqvist, P., Chevalier, R. A., \& Kirshner, R. P. 1995, ApJ, 439, 730-751

Potter, T. M., Staveley-Smith, L., Ng, C. Y., Ball, L., Gaensler, B. M., Kesteven, M. J., Manchester, R. N. Tzioumis, A. K., \& Zanardo, G. 2009, ApJ, 705, 261-271

Sutherland, R. S. \& Bicknell, G. V. 2007, ApJS, 173, 37-69

Truelove, J. K. \& McKee, C. F. 1999, ApJS, 120, 299-236

Zhekov, S. A. Park, S., McCray, R., Racusin, J. L., \& Burrows, D. N. 2010, MNRAS, 407, $1157-1169$ 